\title{
Acute two-vessel occlusion due to simultaneous very late stent thrombosis following sirolimus-eluting stent implantation: a case report and review of the literature
}

\author{
Zhenchi Sang, Huigen Jin, Zongjun Liu \\ Department of Cardiology, Pu Tuo Hospital, Shanghai University of Traditional Chinese Medicine, Shanghai, China
}

Postep Kardiol Inter 2015; 11, 3 (41): 252-255

DOI: $10.5114 /$ pwki.2015.54028

\section{Introduction}

Very late stent thrombosis (VLST; $>1$ year) is a rare but fatal complication due to acute vessel closure. Several factors, including procedure, lesion and patient discontinuation of antiplatelet therapy, are most closely related to its occurrence [1]. Furthermore, neoatherosclerotic plaque rupture is now acknowledged as a potential contributing factor [2]. Although similar rates of early and late stent thrombosis were observed between drug-eluting stent (DES) and bare metal stent (BMS) [3]. Very late stent thrombosis occurs with higher frequency in DES [4]. However, it is even rarer to have a simultaneous two-vessel very late stent thrombosis with a sirolimus-eluting stent (SES), and studies on the pathogeny are lacking.

We report the case of a patient presenting with ST-segment-elevation myocardial infarction (STEMI) and cardiogenic shock who experienced simultaneous VLST in two vessels which occurred 40 months after sirolimus-eluting stent implantation.

\section{Case report}

A 56-year-old man presented to the emergency department with severe chest pain within $4 \mathrm{~h}$ after onset of symptoms. The ECG showed an ST-segment elevation in I, aVL and V6-V9 (Figure 1). The patient had undergone percutaneous coronary intervention $(\mathrm{PCl})$ in our catheter lab using sirolimus-eluting stents (Firebird, MicroPort) in the left anterior descending (LAD) $(3.0 \times 33 \mathrm{~mm} ; 16 \mathrm{~atm})$ and in the left circumflex artery $(\mathrm{LCX})(2.75 \times 33 \mathrm{~mm}$; $10 \mathrm{~atm}) 40$ months prior to admission. Also, 35 months prior to admission, an intervention in a de novo lesion of the right coronary artery (RCA) using an SES (Firebird, MicroPort; $4.0 \times 23 \mathrm{~mm}$; $9 \mathrm{~atm}$ ) followed by balloon angioplasty was performed. At this point in time, no evi- dence of restenosis was found in the former lesion of the LAD or LCX. Anti-platelet therapy consisting of $100 \mathrm{mg}$ aspirin and $75 \mathrm{mg}$ clopidogrel was prescribed for an intended period of 12 months following percutaneous intervention.

Cardiac catheterization revealed a thrombotic occlusion at the site of the stent implanted in the LAD as well as at the site of the stent in the LCX (Figure $2 \mathrm{~A}$ ). A temporary pacemaker was inserted, then crossing the lesions of LCX with a guidewire, thrombus aspiration was performed using a thrombus aspiration device (Thrombuster II, KANEKA) starting in the LCX. Intracoronary abciximab followed by intravenous infusion was administered. The angiographic result showed Thrombolysis In Myocardial Infarction (TIMI) 3 flow in the vessel. During the procedure, the patient had cardiac arrest due to ventricular fibrillation (VF). After defibrillation with $360 \mathrm{~J}$, sinus rhythm was restored.

We proceeded with $\mathrm{PCI}$ for the $\mathrm{LAD}$ lesion, the proximal LAD lesion was crossed with a wire and predilated with a $2.5 \times 20 \mathrm{~mm}$ balloon (Sprinter Legend, Medtronic; $8 \mathrm{~atm}$ ), and we deployed a $3.0 \times 23 \mathrm{~mm}$ Firebird at $14 \mathrm{~atm}$ of pressure for $10 \mathrm{~s}$ with a good angiographic result. The patient was kept on an intra-aortic balloon pump overnight.

During the patient's hospitalization, we performed intravascular ultrasound (IVUS) to further evaluate the cause of thrombus. The IVUS (iLab, Boston Scientific, USA) of the lesions demonstrated late stent malapposition, with positive coronary vessel remodeling around the stent in LAD and LCX (Figure 3). Subsequently, we used a $3.0 \times 10 \mathrm{~mm}$ balloon (Hiryu, Terumo; $16 \mathrm{~atm}$ ) to postdilate the stent and deployed a $3.0 \times 18 \mathrm{~mm}$ Firebird stent at $16 \mathrm{~atm}$ of pressure to cover the previous stent in LCX,

\section{Corresponding author:}

Zhenchi Sang, Department of Cardiology, Pu Tuo Hospital, Shanghai University of Traditional Chinese Medicine, 164 LanXi Road, 200062 Shanghai, China, phone: +86 021 62572723, e-mail: sangzc@126.com

Received: 10.03 .2015 , accepted: 14.03 .2015$. 


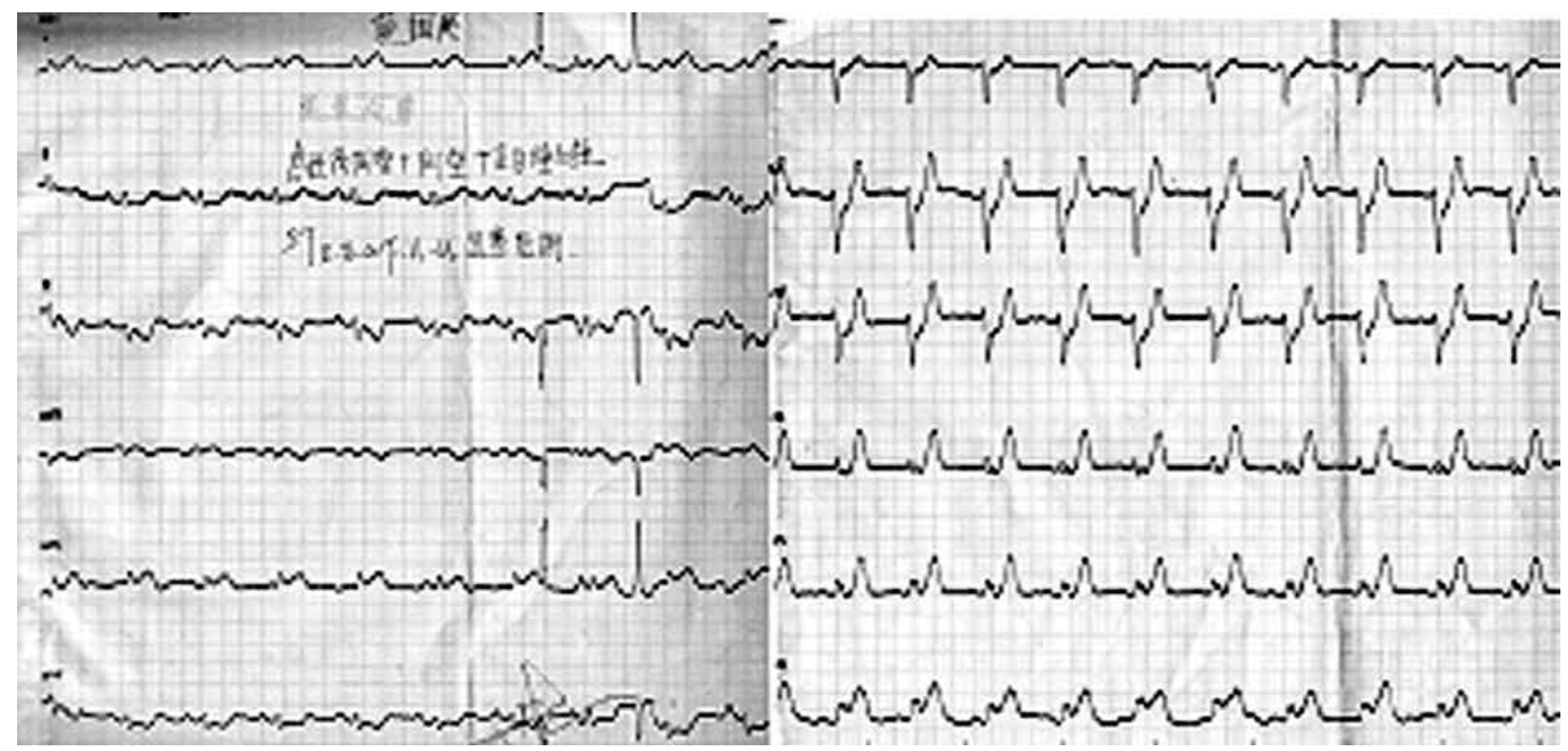

Figure 1. ECG at time of admission
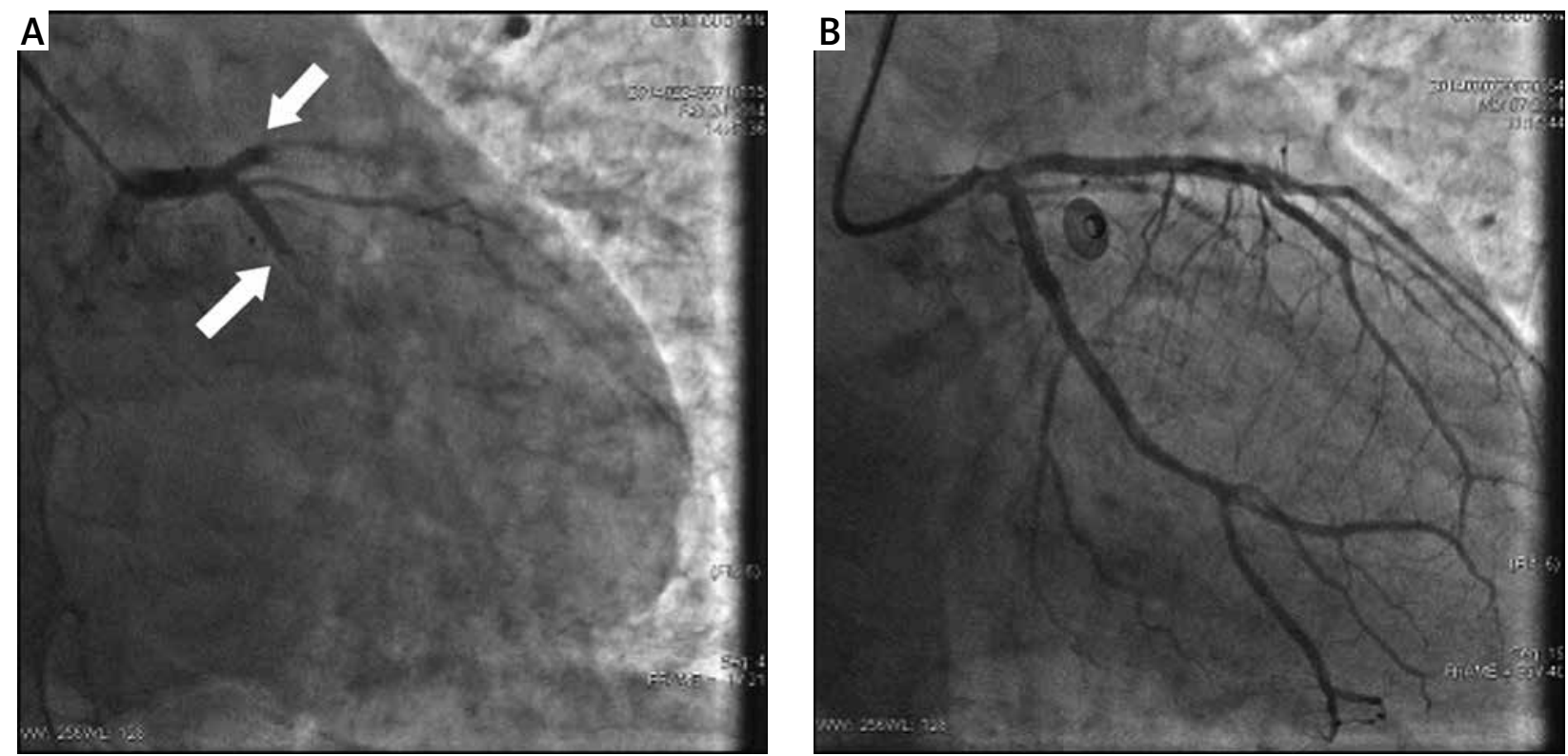

Figure 2. A - Coronary angiogram showed the left anterior descending and left circumflex artery thrombotic mid stent occlusion (white arrow). B - Coronary angiogram showed no residual stenosis and relatively good distal flow in two vessels

giving a good end result (Figure 2 B). The patient was rehospitalized for heart failure 1 month after discharge.

\section{Discussion and review of the literature}

The incidence of VLST appears to be between $0.4 \%$ and $0.6 \%$ per year in the literature [5], which can lead to high mortality [6]. The mechanism is not fully understood. Delayed endothelialization and polymer induced inflammation [7], stent fracture [8], late stent malapposition, stent underexpansion [9], incomplete neointimal coverage over stent struts, and neoatherosclerotic plaque rupture [2] may play a vital role in the progression of
VLST. To the best of our knowledge, this is the first reported case of simultaneously VLST occurring at the site of stents in LAD and LCX 40 months after SES implantation in Chinese patients. Late acquired incomplete stent apposition (LAISA) was identified by IVUS.

Previous studies demonstrated that VLST occurred more frequently in DES than in BMS [4]. Togni et al. [10] reported that paradoxical exercise-induced vasoconstriction occurred in both proximal and distal segments of the vessel adjacent to SESs but did not occur in the BMS vessel. Drug-induced endothelial dysfunction was considered a contributor to VLST. Inoue et al. [11] found 

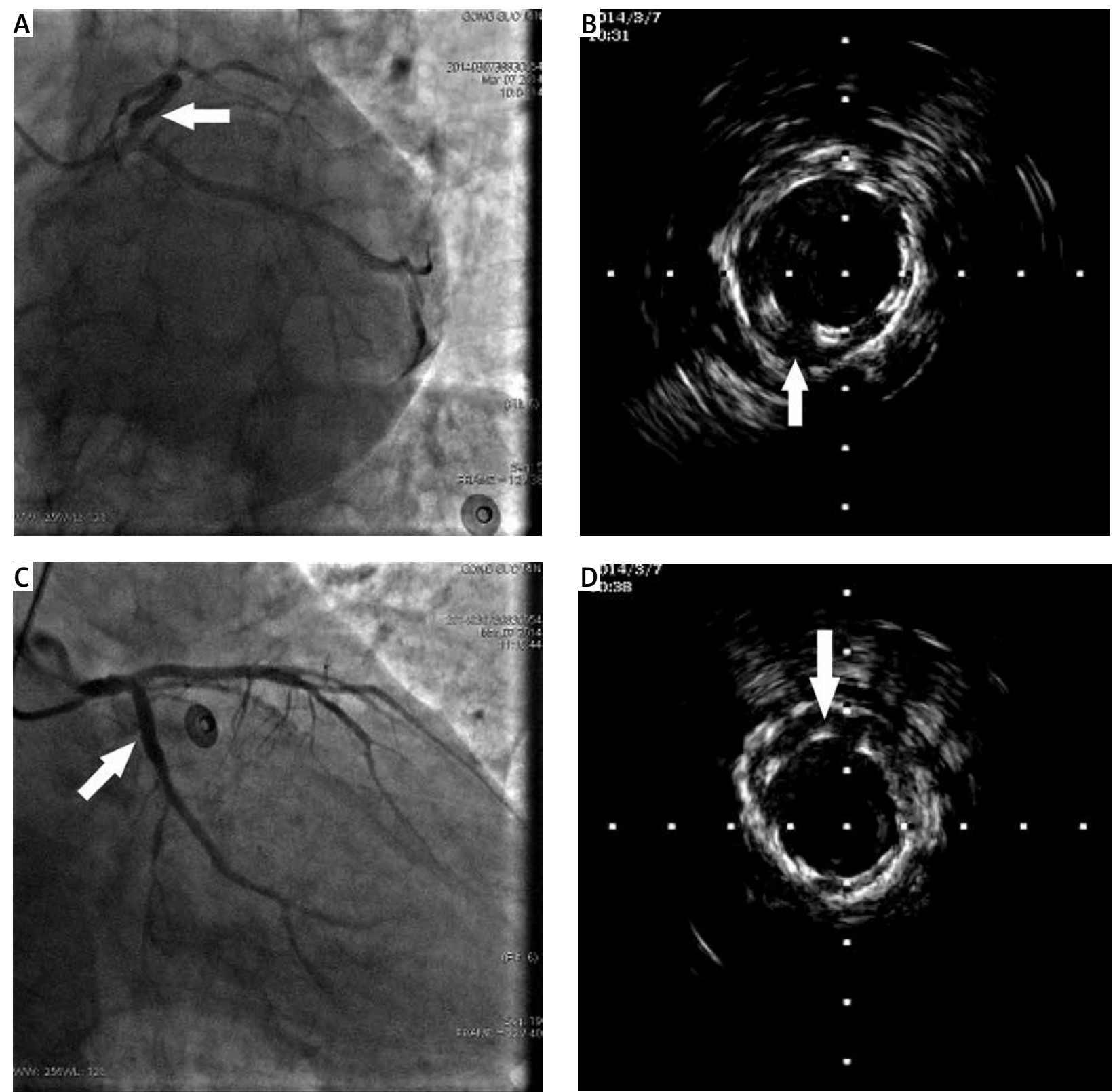

Figure 3. A - Coronary angiography showed peri-stent contrast staining at the site of LAD DES (white arrow). B - IVUS showed late stent malapposition in the LAD (white arrow). C - Coronary angiography showed peristent contrast staining at the site of LCX DES (white arrow). D - IVUS showed late stent malapposition in the LCX (white arrow)

fibrin deposition and infiltration of chronic inflammatory cells around the SES struts, which might be followed by VLST. Furthermore, Caixeta et al. also reported a case of VLST in a BMS induced by a severe malapposition and underexpansion [9]. This suggests that besides the drug, other factors can also cause VLST.

Nakazawa et al. [12] studied the coronary responses and differential mechanisms of VLST attributed to SES and paclitaxel-eluting stent (PES). In this study, the incidence of VLST did not differ significantly between SES and PES ( $21 \%$ vs. $27 \%, p=0.47)$. But for SES, localized hypersensitivity reactions and inflammation which result in positive remodeling of the vessel and malapposition are the primary contributors. In contrast, VLST in PES was attributed to malapposition secondary to excessive fibrin deposition on the abluminal surface. Recently Yamawaki et al. [13] reported a case of simultaneous VLST of both LAD and LCX after implantation of two SESs. Intravascular ultrasound showed LAISA of two vessels. But in the RCA which was implanted with PES, IVUS did not show such a finding. The IVUS finding demonstrated "different vascular reactions" to "different types of DES".

Reported cases of VLST occurring in second-generation DES in the literature are rare. Puri et al. [14] report- 
ed a case of simultaneous two-vessel VLST after everolimus-eluting stent (EES) implantation. Intravascular ultrasound showed stent malapposition with positive remodeling of the vessel wall. A previous study [15] indicated that the incidence of late acquired Peri-stent contrast staining (PSS) with EES was lower than that with SES. Peri-stent contrast staining has been reported to be associated with VLST. However, it remains to be determined whether there is a difference between EES and SES in the occurrence of VLST.

A previous report [16] indicated two possible mechanisms contributing to LAISA: regional positive remodeling with an increase in vessel area out of proportion to the increase in plaque area; or thrombus dissolution in the case of primary angioplasty. The potential therapeutic effect associated with correction of late incomplete stent apposition is unknown, and whether prolonged dual antiplatelet therapy can decrease the incidence of VLST is also uncertain.

\section{Conclusions}

This case report illustrates mechanisms of VLST after stent implantation. Late acquired incomplete stent apposition played a significant role after SES implantation. The occurrence of VLST in different types of stent, including BMS, SES, PES and EES, varied. Angiography with intravascular ultrasound can be used to identify VLST and LAISA.

\section{Conflict of interest}

The authors declare no conflict of interest.

\section{References}

1. Baran KW, Lasala JM, Cox DA, et al. A clinical risk score for the prediction of very late stent thrombosis in drug eluting stent patients. Eurolntervention 2011; 6: 949-54.

2. Amabile N, Souteyrand G, Ghostine S, et al. Very late stent thrombosis related to incomplete neointimal coverage or neoatherosclerotic plaque rupture identified by optical coherence tomography imaging. Eur Heart J Cardiovasc Imaging 2014; 15 : 24-31.

3. Dangas GD, Caixeta A, Mehran R, et al. Harmonizing Outcomes With Revascularization and Stents in Acute Myocardial Infarction (HORIZONS-AMI) Trial Investigators. Frequency and predictors of stent thrombosis after percutaneous coronary intervention in acute myocardial infarction. Circulation 2011; 123: 1745-56.

4. Jensen LO, Maeng M, Kaltoft A, et al. Stent thrombosis, myocardial infarction, and death after drug-eluting and bare-metal stent coronary interventions. J Am Coll Cardiol 2007; 50: 463-70.

5. Daemen J, Wenaweser P, Tsuchida K, et al. Early and late coronary stent thrombosis of sirolimus eluting and paclitaxel-eluting stents in routine clinical practice: data from a large two-institutional cohort study. Lancet 2007; 369: 667-78.

6. Kubler P, Reczuch K. The use of drug-eluting stents in acute myocardial infarction - is the battle coming to an end? From despair to acceptance. Postep Kardiol Inter 2013; 9: 50-4.
7. Azarbal B, Currier JW. Allergic reactions after the implantation of drug-eluting stents: is it the pill or the polymer? J Am Coll Cardiol 2006; 47: 182-3.

8. Lee SW, Tam FC, Chan KK. Very late stent thrombosis due to DES fracture: description of a case and review of potential causes. Catheter Cardiovasc Interv 2011; 78: 1101-5.

9. Caixeta A, Braga VC, Mintz GS. Very late stent thrombosis with bare-metal stent: identifying severe stent malapposition and underexpansion by intravascular ultrasound. Einstein 2013; 11: 364-6.

10. Togni M, Windecker S, Cocchia R, et al. Sirolimus-eluting stents associated with paradoxic coronary vasoconstriction. J Am Coll Cardiol 2005; 46: 231-6.

11. Inoue K, Mitsudo K, Nobuyoshi M. Abstract 4724: Pathological mechanisms of very late stent thrombosis after drug-eluting stent implantation in human coronary arteries [abstract]. Circulation 2009; 120 (18 Suppl 2): S993.

12. Nakazawa G, Finn AV, Vorpahl M, et al. Coronary responses and diferential mechanisms of late stent thrombosis attributed to first-generation sirolimus- and paclitaxel-eluting stents. J Am Coll Cardiol 2011; 57: 390-8.

13. Yamawaki M, Onuma Y, Nakano M, et al. Simultaneous occlusion of left anterior descending and left circumflex arteries by very late stent thrombosis: vascular response to drug-eluting stents assessed by intravascular ultrasound. Heart Vessels 2014 Jul 17 [Epub ahead of print].

14. Puri A, Saireddy R, McClean D. Simultaneous two-vessel very late stent thrombosis of everolimus-eluting stents. J Invasive Cardiol 2013; 25: E48-50.

15. Fujiwara T, Sakakura K, Ako J, et al. Occurrence of late acquired peristent contrast staining: comparison between sirolimus-eluting stents and everolimus-eluting stents. Int Heart J 2012; 53: 165-9.

16. Sgueglia GA, Stipo A, Pucci E. Late acquired incomplete stent apposition. Arch Cardiovasc Dis 2013; 106: 240-2. 